\title{
Inflammation: a Good Research Target to Improve Outcomes of Poor-Grade Subarachnoid Hemorrhage
}

\author{
Hidenori Suzuki ${ }^{1}$ (D) \\ Received: 6 June 2019 / Revised: 6 June 2019 / Accepted: 11 June 2019/Published online: 18 June 2019 \\ (C) Springer Science+Business Media, LLC, part of Springer Nature 2019
}

Aneurysmal subarachnoid hemorrhage (SAH) remains a devastating cerebrovascular disease [1] and should be treated as a systemic disorder needing intensive care, not merely as an intracranial disorder, especially in poor-grade SAH patients [2]. Aging and poorer admission clinical grade are the most important predictors of poor outcomes [3]. In our recent series treated with early aneurysmal obliteration by endovascular coiling or surgical clipping, SAH patients over age 75 had 3month modified Rankin scales 3-6 (poor outcomes) in 73.1\% of cases with admission World Federation of Neurological Surgeons (WFNS) grades I-III and in $77.4 \%$ of cases with admission WFNS grades IV-V (poor grade), while SAH patients under age 75 had 3-month poor outcomes only in $13.1 \%$ of cases with admission WFNS grades I-III but still in 59.3\% of cases with admission WFNS grades IV-V (unpublished data). All acute SAH patients with $>10 \mathrm{~mm}^{2}$ ischemic brain lesion on diffusion-weighted magnetic resonance imaging or prolonged mean transit time $>6.385 \mathrm{~s}$ in the whole brain on computed tomography perfusion may have irreversible brain damage and resulted in poor outcomes irrespective of any treatment including cerebrospinal fluid drainage and decompressive craniectomy to control intracranial pressure in addition to early aneurysmal obliteration $[4,5]$. The other patients with WFNS grade $\mathrm{V}$ had poor outcomes in $70-80 \%$, even though the maximal treatment was undergone [4-6]; however, it should be noticed that no treatment caused poor outcomes in all grade $\mathrm{V}$ patients [6]. There are poor evidence and no guidelines available for the treatment of poor-grade SAH patients.

From a basic research point of view, admission poor clinical grade indicates that severe early brain injury (EBI) is arising at admission and possibly leads to poor outcomes directly or associated with delayed cerebral ischemia [1].

Hidenori Suzuki

mie1192suzuki@gmail.com

1 Department of Neurosurgery, Mie University Graduate School of Medicine, 2-174 Edobashi, Tsu, Mie 514-8507, Japan
Although EBI is multifactorial and includes multiple pathologies including neuroinflammation [7], oxidative stress [8], neuronal apoptosis [9], blood-brain barrier disruption [10], microvascular constriction, and microthrombi [11], the pathologies may interact with each other. Delayed cerebral ischemia may be caused by cerebral vasospasm, cortical spreading depolarization, and microcirculatory dysfunction [12], which may occur secondary to EBI [13]. Although cerebral vasospasm remains an important cause of delayed cerebral ischemia [14], microcirculatory disturbance and cortical spreading depolarization may be more important than cerebral vasospasm as to the impact on functional outcomes especially in poor-grade SAH patients [15]. Thus, many basic researchers have worked hard to overcome EBI, which is believed eventually to improve outcomes of poor-grade SAH patients.

It is no doubt that an abrupt elevation of intracranial pressure by a rupture of cerebral aneurysm is an essential trigger of the development of EBI. Even though the amount and distribution of blood in the subarachnoid space are similar, an abrupt increase in intracranial pressure disrupts cerebral perfusion associated with early, pronounced, and sustained disruption of cerebral autoregulation as well as neuronal damage, possibly leading to more severe EBI and poorer outcomes [16]. Severe SAH induced glial and endothelial injuries contributing to increased blood-brain barrier permeability immediately after SAH by endovascular perforation in rats [17]. Severe SAH-induced intracranial pressure elevation and brain injury also activate the sympathetic nervous system, release catecholamines, and induce systemic inflammation and extracerebral organs injury [18]. In endovascular perforation SAH models using adhesion molecule knockout mice, it was demonstrated that SAH resulted in early intravascular inflammation with the recruitment of circulating neutrophils to the vessel/brain interface and that the inhibition of neutrophilendothelial interaction prevented systemic inflammation from accumulating and activating microglia to cause neuronal cell death [19]. Extravasated blood components such as thrombin, platelets, and leukocytes also activate microglia, which is an 
important mechanism to produce proinflammatory cytokines, matricellular proteins, and oxidative stress, causing EBI in a mouse endovascular perforation model [20, 21]. At $24 \mathrm{~h}$ after experimental SAH by endovascular perforation in rats, magnetic resonance imaging studies showed that more severe SAH caused a more frequent and larger cerebral infarction, where fibrinogen/fibrin-stained microthromboses were histologically found [22]. Thus, although several challenges exist to translate findings from animal research to clinical settings [23], the endovascular perforation model associated with arterial bleeding may be the most suitable to study severe EBI, and in fact it has the highest mortality among SAH models in mice [24]. As to target pathologies, inflammation may be a good candidate [21]: to put it more concretely, damageassociated molecular patterns (DAMPs) and pattern recognition receptors may be important initiators as well as enhancers of post-SAH neuroinflammation leading to severe EBI [1].

Inflammation under sterile conditions is a critical response to tissue injuries and is induced by the release of endogenous molecules called DAMPs [25]. Although DAMP is hidden by compartmentalization within cells or sequestration in the extracellular matrix under normal conditions, DAMP is released from any injured cell (plasma membrane, cytosol, mitochondria, endoplasmic reticulum, nucleus, or autophagosomederived DAMPs) and proteolytically from the extracellular matrix undergoing stresses or injuries (extracellular matrixderived DMAPs), and is recognized by pattern recognition receptors $[25,26]$. Currently, 5 classes of pattern recognition receptors are known as follows: cell surface or endosomal Toll-like receptors (TLRs), cytoplasmic nucleotide-binding oligomerization domain-like receptors and inflammasome, intracellular retinoic acid-inducible gene-I-like receptors, transmembrane C-type lectin receptors, and absent in melanoma 2like receptors [25]. Of the 11 human and 13 murine TLR family members, TLR4 can mediate the strongest inflammatory reaction via both the myeloid differentiation primaryresponse protein 88-dependent and the Toll receptorassociated activator of interferon-dependent cascades, and is expressed on various cells including microglia, neurons, astrocytes, brain capillary endothelial cells, endothelial and smooth muscle cells of cerebral arteries, and peripheral blood cells such as leukocytes, macrophages, and platelets [27]. In addition, TLR4 recognizes many DAMPs derived from plasma membrane (syndecans and glypicans), cytosol (S100 proteins [S100B, S100A2], heat shock proteins, heme, oxyhemoglobin, methemoglobin, and hemin), nucleus (high mobility group box-1 and histones), autophagosome (high mobility group box-1), and extracellular matrix (extravasation [fibrinogen and fibrin], proteoglycan [biglycan and decorin], glycosaminoglycan [hyaluronan and heparan sulfate], glycoprotein [fibronectin], and matricellular proteins [tenascin-C and galectin-3]); and TLR4 may be the only pattern recognition receptor binding to extravasated blood components $[25,26]$.
Glycosaminoglycans such as heparin sulfate and hyaluronic acid account for a major part of the extracellular matrix in the central nervous system and are important mediators in multiple functions during the development, physiology, and pathology of the central nervous system [28], but matricellular proteins are increased by de novo synthesis [29]. Heat shock proteins respond to a wide variety of brain injuries including ischemic and hemorrhagic strokes, and can be protective or harmful to the brain [30]. Taken together, TLR4 and/or de novo synthetics matricellular proteins may be good therapeutic targets to inhibit neuroinflammation after SAH. At aneurysmal rupture, the resultant more tissue injuries and extravasated blood activate more TLR4, which is an initial step of cascades to neuroinflammation and more induces matricellular proteins, chemokines, adhesion molecules, proinflammatory cytokines, and mediators, causing severe EBI and delayed cerebral ischemia [27]. As some of the matricellular proteins are DAMPs and ligands of TLR4, TLR4-matricellular proteins-TLR4 signaling may form a vicious circle leading to more severe EBI and delayed cerebral ischemia [31]. However, as various pathophysiological reactions other than EBI and delayed cerebral ischemia affect inflammatory reactions, therefore, the interactions cause low specificity of proinflammatory cytokines or mediators other than matricellular proteins in EBI and delayed cerebral ischemia, although neuroinflammation is a major aspect of EBI and delayed cerebral ischemia [1]. Now, the findings indicating the linkage between TLR4 $[31,32]$ or matricellular proteins (tenascin-C [33-35] and galectin-3 [36, 37]) and EBI or delayed cerebral ischemia are increasing experimentally and clinically. A local environment such as extracellular $\mathrm{pH}$ is reported to affect tenascin-C's function [1,38], and the functional relationships between tenascin- $\mathrm{C}$ and other matricellular proteins such as galectin-3 [1], periostin [39-41], and osteopontin [42, 43] should be also investigated in EBI and delayed cerebral ischemia. An antiplatelet cilostazol inhibited post-SAH tenascin-C induction [44] and improved outcomes associated with reduced incidence of delayed cerebral infarction in a clinical setting [3].

Another potential therapeutic target is cellular microparticles, which are small vesicles formed by plasma membranes of various vascular cells such as endothelial cells, smooth muscle cells, platelets, leukocytes, and erythrocytes [45]. Cellular microparticles are released from the membranes by triggers causing cellular activation or apoptosis including cytokines, hypoxia and shear stress, and transfer receptors and cytosolic contents such as enzymes, ribonucleic, and deoxyribonucleic acids to cells of different origin [45, 46]. In clinical $\mathrm{SAH}$, plasma levels of various cell-derived microparticles, especially tissue factor-expressing and endothelial cellassociated microparticles, were elevated at day 0 post-SAH, and the development of cerebral infarction by day 14, suggesting the linkage among inflammation, endothelial dysfunction, 
and thrombosis, were predicted [47]. Platelet-related systemic thrombogenicity is also reported to be more increased from an acute phase of poorer SAH possibly reflecting systemic inflammatory reaction syndrome, leading to a higher risk of an increased burden of cerebral microinfarcts and delayed cerebral ischemia [48].

All researchers agree that EBI and delayed cerebral ischemia are multifactorial, and that EBI needs to be overcome to improve outcomes of poor-grade SAH. However, current treatments against poor-grade $\mathrm{SAH}$ is somewhat a carpetbombing approach, and the effects are limited. I think that we should develop molecular-targeted drugs against each pathology in EBI to overcome EBI, and that the time is approaching.

Funding This study was funded by a grant-in-aid for Scientific Research from Japan Society for the Promotion of Science (grant number, 17K10825) to Dr. Suzuki.

\section{Compliance with Ethical Standards}

Conflict of Interest The author declares that he has no conflicts of interest.

Ethical Approval This article does not contain any studies with human participants or animals performed by the author.

\section{References}

1. Suzuki H, Fujimoto M, Kawakita F, Liu L, Nakatsuka Y, Nakano F, et al. Tenascin-C in brain injuries and edema after subarachnoid hemorrhage: findings from basic and clinical studies. J Neurosci Res. 2018. https://doi.org/10.1002/jnr.24330.

2. Suzuki H. What is early brain injury? Transl Stroke Res. 2015;6:13 .

3. Suzuki H, Nakatsuka Y, Yasuda R, Shiba M, Miura Y, Terashima $\mathrm{M}$, et al. Dose-dependent inhibitory effects of cilostazol on delayed cerebral infarction after aneurysmal subarachnoid hemorrhage. Transl Stroke Res. 2018. https://doi.org/10.1007/s12975-0180650-y.

4. Sato K, Shimizu H, Fujimura M, Inoue T, Matsumoto Y, Tominaga $\mathrm{T}$. Acute-stage diffusion-weighted magnetic resonance imaging for predicting outcome of poor-grade aneurysmal subarachnoid hemorrhage. J Cereb Blood Flow Metab. 2010;30:1110-20.

5. Sasahara A, Suzuki K, Takahashi Y, Koseki H, Hirota K, Ohbuchi $\mathrm{H}$, et al. Prognostic assessment of aneurysmal subarachnoid patients with WFNS grade V by CT perfusion on arrival. World Neurosurg. 2016;92:1-6.

6. Hoogmoed J, Coert BA, van den Berg R, Roos YBWEM, Horn J, Vandertop WP, et al. Early treatment decisions in poor-grade patients with subarachnoid hemorrhage. World Neurosurg. 2018;119: e568-73.

7. Liu L, Fujimoto M, Nakano F, Nishikawa H, Okada T, Kawakita F, et al. Deficiency of tenascin-C alleviates neuronal apoptosis and neuroinflammation after experimental subarachnoid hemorrhage in mice. Mol Neurobiol. 2018;55:8346-54.

8. Fumoto T, Naraoka M, Katagai T, Li Y, Shimamura N, Ohkuma H. The role of oxidative stress in microvascular disturbances after experimental subarachnoid hemorrhage. Transl Stroke Res. 2019. https://doi.org/10.1007/s12975-018-0685-0.
9. Shi L, Al-Baadani A, Zhou K, Shao A, Xu S, Chen S, et al. PCMT1 ameliorates neuronal apoptosis by inhibiting the activation of MST1 after subarachnoid hemorrhage in rats. Transl Stroke Res. 2017;8:474-83.

10. Kanamaru H, Suzuki H. Potential therapeutic molecular targets for blood-brain barrier disruption after subarachnoid hemorrhage. Neural Regen Res. 2019;14:1138-43.

11. Tso MK, Macdonald RL. Subarachnoid hemorrhage: a review of experimental studies on the microcirculation and the neurovascular unit. Transl Stroke Res. 2014;5:174-89.

12. Brathwaite S, Macdonald RL. Current management of delayed cerebral ischemia: update from results of recent clinical trials. Transl Stroke Res. 2014;5:207-26.

13. Fujii M, Yan J, Rolland WB, Soejima Y, Caner B, Zhang JH. Early brain injury, an evolving frontier in subarachnoid hemorrhage research. Transl Stroke Res. 2013;4:432-46.

14. Nakano F, Kawakita F, Liu L, Nakatsuka Y, Nishikawa H, Okada T, et al. Anti-vasospastic effects of epidermal growth factor receptor inhibitors after subarachnoid hemorrhage in mice. Mol Neurobiol. 2018. https://doi.org/10.1007/s12035-018-1400-6.

15. Neulen A, Meyer S, Kramer A, Pantel T, Kosterhon M, Kunzelmann S, et al. Large vessel vasospasm is not associated with cerebral cortical hypoperfusion in a murine model of subarachnoid hemorrhage. Transl Stroke Res. 2019;10:319-26.

16. Conzen C, Becker K, Albanna W, Weiss M, Bach A, Lushina N, et al. The acute phase of experimental subarachnoid hemorrhage: intracranial pressure dynamics and their effect on cerebral blood flow and autoregulation. Transl Stroke Res. 2018. https://doi.org/ 10.1007/s12975-018-0674-3.

17. Hasegawa Y, Suzuki H, Uekawa K, Kawano T, Kim-Mitsuyama S. Characteristics of cerebrovascular injury in the hyperacute phase after induced severe subarachnoid hemorrhage. Transl Stroke Res. 2015;6:458-66.

18. Chen S, Li Q, Wu H, Krafft PR, Wang Z, Zhang JH. The harmful effects of subarachnoid hemorrhage on extracerebral organs. Biomed Res Int. 2014;2014(858496):1-12. https://doi.org/10. 1155/2014/858496.

19. Atangana E, Schneider UC, Blecharz K, Magrini S, Wagner J, Nieminen-Kelhä $\mathrm{M}$, et al. Intravascular inflammation triggers intracerebral activated microglia and contributes to secondary brain injury after experimental subarachnoid hemorrhage (eSAH). Transl Stroke Res. 2017;8:144-56.

20. Pang J, Peng J, Matei N, Yang P, Kuai L, Wu Y, et al. Apolipoprotein E exerts a whole-brain protective property by promoting M1? Microglia quiescence after experimental subarachnoid hemorrhage in mice. Transl Stroke Res. 2018;9:654-68.

21. Nishikawa H, Suzuki H. Possible role of inflammation and galectin3 in brain injury after subarachnoid hemorrhage. Brain Sci 2018;8. doi https://doi.org/10.3390/brainsci8020030.

22. Guo D, Wilkinson DA, Thompson BG, Pandey AS, Keep RF, Xi G, et al. MRI characterization in the acute phase of experimental subarachnoid hemorrhage. Transl Stroke Res. 2017;8:234-43.

23. Suzuki H, Nakano F. To improve translational research in subarachnoid hemorrhage. Transl Stroke Res. 2018;9:1-3.

24. Kamp MA, van Lieshout JH, Dibué-Adjei M, Weber JK, Schneider $\mathrm{T}$, Restin $\mathrm{T}$, et al. A systematic and meta-analysis of mortality in experimental mouse models analyzing delayed cerebral ischemia after subarachnoid hemorrhage. Transl Stroke Res. 2017;8:206-19.

25. Schaefer L. Complexity of danger: the diverse nature of damageassociated molecular patterns. J Biol Chem. 2014;289:35237-45.

26. Chaudhry SR, Hafez A, Jahromi BR, Kinfe TM, Lamprecht A, Niemelä M, Muhammad S. Role of damage associated molecular pattern molecules (DAMPs) in aneurysmal subarachnoid hemorrhage (aSAH). Int J Mol Sci 2018;19. doi https://doi.org/10.3390/ ijms19072035. 
27. Okada T, Suzuki H. Toll-like receptor 4 as a possible therapeutic target for delayed brain injuries after aneurysmal subarachnoid hemorrhage. Neural Regen Res. 2017;12:193-6.

28. Lim TC, Spector M. Biomaterials for enhancing CNS repair. Transl Stroke Res. 2017:8:57-64.

29. Suzuki H, Nishikawa H, Kawakita F. Matricellular proteins as possible biomarkers for early brain injury after aneurysmal subarachnoid hemorrhage. Neural Regen Res. 2018;13:1175-8.

30. Sharp FR, Zhan X, Liu D-Z. Heat shock proteins in the brain: role of Hsp70, Hsp 27, and HO-1 (Hsp32) and their therapeutic potential. Transl Stroke Res. 2013;4:685-92.

31. Okada T, Kawakita F, Nishikawa H, Nakano F, Liu L, Suzuki H. Selective Toll-like receptor 4 antagonists prevent acute blood-brain barrier disruption after subarachnoid hemorrhage in mice. Mol Neurobiol. 2019;56:976-85.

32. Kawakita F, Fujimoto M, Liu L, Nakano F, Nakatsuka Y, Suzuki H. Effects of Toll-like receptor 4 antagonists against cerebral vasospasm after experimental subarachnoid hemorrhage in mice. Mol Neurobiol. 2017;54:6624-33.

33. Fujimoto M, Shiba M, Kawakita F, Liu L, Shimojo N, ImanakaYoshida K, et al. Effects of tenascin-C knockout on cerebral vasospasm after experimental subarachnoid hemorrhage in mice. Mol Neurobiol. 2018;55:1951-8.

34. Shiba M, Suzuki H. Lessons from tenascin-C knockout mice and potential clinical application to subarachnoid hemorrhage. Neural Regen Res. 2019;14:262-4.

35. Shiba M, Fujimoto M, Imanaka-Yoshida K, Yoshida T, Taki W, Suzuki H. Tenascin-C causes neuronal apoptosis after subarachnoid hemorrhage in rats. Transl Stroke Res. 2014;5:238-47.

36. Nishikawa H, Nakatsuka Y, Shiba M, Kawakita F, Fujimoto M, Suzuki H. Increased plasma galectin-3 preceding the development of delayed cerebral infarction and eventual poor outcome in nonsevere aneurysmal subarachnoid hemorrhage. Transl Stroke Res. 2018;9:110-9.

37. Nishikawa H, Liu L, Nakano F, Kawakita F, Kanamaru H, Nakatsuka Y, et al. Modified citrus pectin prevents blood-brain barrier disruption in mouse subarachnoid hemorrhage by inhibiting galectin-3. Stroke. 2018;49:2743-51.

38. Suzuki H, Shiba M, Nakatsuka Y, Nakano F, Nishikawa H. Higher cerebrospinal fluid $\mathrm{pH}$ may contribute to the development of delayed cerebral ischemia after aneurysmal subarachnoid hemorrhage. Transl Stroke Res. 2017;8:165-73.
39. Liu L, Kawakita F, Fujimoto M, Nakano F, Imanaka-Yoshida K, Yoshida T, et al. Role of periostin in early brain injury after subarachnoid hemorrhage in mice. Stroke. 2017;48:1108-11.

40. Nishikawa H, Suzuki H. Implications of periostin in the development of subarachnoid hemorrhage-induced brain injuries. Neural Regen Res. 2017;12:1982-4.

41. Kanamaru H, Kawakita F, Nakano F, Miura Y, Shiba M, Yasuda R, et al. pSEED groupPlasma periostin and delayed cerebral ischemia after aneurysmal subarachnoid hemorrhage. Neurotherapeutics. 2019;16:480-90. https://doi.org/10.1007/s13311-018-00707-y.

42. Tanioka S, Ishida F, Nakano F, Kawakita F, Kanamaru H, Nakatsuka Y, Nishikawa H, Suzuki H; pSEED group. Machine learning analysis of matricellular proteins and clinical variables for early prediction of delayed cerebral ischemia after aneurysmal subarachnoid hemorrhage. Mol Neurobiol. 2019. doi https://doi. org/10.1007/s12035-019-1601-7.

43. Nakatsuka Y, Shiba M, Nishikawa H, Terashima M, Kawakita F, Fujimoto M, et al. Acute-phase plasma osteopontin as an independent predictor for poor outcome after aneurysmal subarachnoid hemorrhage. Mol Neurobiol. 2018;55:6841-9.

44. Nakatsuka Y, Kawakita F, Yasuda R, Umeda Y, Toma N, Sakaida $\mathrm{H}$, et al. Preventive effects of cilostazol against the development of shunt-dependent hydrocephalus after subarachnoid hemorrhage. J Neurosurg. 2017;127:319-26.

45. Boettinger S, Lackner P. Cellular microparticles in subarachnoid hemorrhage. Transl Stroke Res. 2015;6:342-4.

46. VanWijk MJ, VanBavel E, Sturk A, Nieuwland R. Microparticles in cardiovascular diseases. Cardiovasc Res. 2003;59:277-87.

47. Sanborn MR, Thom SR, Bohman LE, Stein SC, Levine JM, Milovanova T, et al. Temporal dynamics of microparticle elevation following subarachnoid hemorrhage. J Neurosurg. 2012;117:57986.

48. Ray B, Pandav VM, Mathews EA, Thompson DM, Ford L, Yearout LK, et al. Coated-platelet trends predict short-term clinical outcome after subarachnoid hemorrhage. Transl Stroke Res. 2018;9:459-70.

Publisher's Note Springer Nature remains neutral with regard to jurisdictional claims in published maps and institutional affiliations. 